\title{
PULSED GAMMA-RAY EMISSION FROM SHORT-PERIOD PULSARS
}

\author{
K. S. Cheng and Winnis K. Y. Ding \\ Department of Physics, University of Hong Kong, Pokfulam Road, Hong Kong \\ Received 1993 August 31; accepted 1994 February 24
}

\begin{abstract}
We study the gamma-ray emission mechanisms from pulsars with period, $P$, between $4.6 \times 10^{-2} B_{12}^{2 / 5} \mathrm{~s}$ and $0.17 B^{5 / 12} \sin ^{1 / 6} \theta \alpha^{-5 / 4} \mathrm{~s}$ in terms of outermagnetospheric gap model, in which electrostatic accelerators have been proposed to exist in regions near the null charge surfaces where $\boldsymbol{\Omega} \cdot \boldsymbol{B}=0$. We found that the spectra of all known $\gamma$-ray pulsars can be fitted by two free parameters, namely, $\alpha r_{L}$, the mean distance to the outergap, and $\sin \theta$, the mean pitch angle of the secondary $e^{ \pm}$pairs. Gamma-rays from those pulsars with $P<0.17 B^{5 / 12} \sin ^{1 / 6} \theta \alpha^{-5 / 4}$ are mainly emitted by secondary $e^{ \pm}$pairs, which are created beyond the outergap, via synchrotron radiation and the gamma-ray emission efficiency is $\sim 10^{-2}$. For pulsars with period approaching $\sim 0.17 B^{5 / 12} \sin ^{1 / 6} \theta \alpha^{-5 / 4} \mathrm{~s}$, their gamma-ray emission efficiency is approaching unity. The comparison of model results and the observed data, including gamma-ray spectrum, efficiency, relative intensity, and phase separation of pulses are discussed in the text.
\end{abstract}

Subject headings: gamma rays: theory - pulsars: general - radiation mechanisms: nonthermal

\section{INTRODUCTION}

Theoretically, $\gamma$-rays are always associated with the radio emission of pulsar (Goldreich \& Julian 1969; Ruderman \& Sutherland 1975). It is widely accepted that electrostatic acceleration regions, where the electric field along the stellar magnetic field is not zero, exist above the polar caps of pulsar (polar gaps). Such an electric field can accelerate $e^{-} / e^{+}$to extremely relativistic speeds and each $e^{-} / e^{+}$can radiate a large number of curvature photons whose typical energies are of the order of a few tens of $\mathrm{MeV}$. These primary photons are sufficiently energetic to produce secondary pairs by local magnetic field (Sturrock 1971). Particle clumping resulting from the two stream instability can radiate coherent radio waves. Therefore, it is no doubt that radio pulsars can emit $\gamma$-rays. The main questions are these:

1. Why are there only six $\gamma$-ray pulsars detected out of over 500 radio pulsars?

2. Why are old pulsars like Geminga and PSR 1055-52 such efficient $\gamma$-ray emitters?

3. Why do the spectra among these six pulsars differ so much?

(Geminga seems to emit most of its spin-down power to photons with energies larger than $10^{2} \mathrm{MeV}$, PSR $1509-58$ emits very few photons with energies higher than a few tens $\mathrm{MeV}$ and the Crab pulsar emits photons from optical to a few $\mathrm{GeV}$. The $\gamma$-ray spectral indices of these five pulsars are ranging from 0.67 to 2.2 .)

Many authors (Bertotti et al. 1969; Sturrock 1971; Treves 1971; Ozernoi \& Usov 1977; Hinata 1977; Harding 1981; Hardee 1979; Ayasli \& Ogelman 1980) have calculated the $\gamma$-ray spectra emitted by the primary and secondary $e^{-}$and/or $e^{+}$from the polar gaps via the mechanism of curvature radiation. The model spectrum rises with an index of $\frac{2}{3}$ and falls of exponentially beyond a critical frequency which completely differs from the spectra of the $\mathrm{Crab}$ and Vela pulsars. More reasonable fit can be obtained by using the mechanism of synchrotron radiation but the model spectrum can only fit the observed spectrum around the turnover portion of the synchrotron spectrum (approximately one decade) and the low energy part is completely off. The total power of the polar gap is limited by the pair production process and the maximum current (Ruderman \& Sutherland 1975) which prevents pulsars lose most of their spin-down energy to $\gamma$-rays via the polar gas.

The outergap models proposed by Cheng, Ho, \& Ruderman (1986a, b, hereafter CHR I, CHR II) discuss a class of rapidly spinning pulsars which can sustain pair production in otherwise charged-depleted regions of the outermagnetosphere (outergaps) near the null charge surface where $\Omega \cdot B=0(\Omega$ is the stellar angular velocity). The charge depletion in regions of the magnetosphere where $\boldsymbol{E} \cdot \boldsymbol{B} \neq 0$ results from the assumed outermagnetospheric current flow along open field lines between the star and the light cylinder. The outgoing current $\left(J_{0}\right)$ is assumed to flow out from the stellar surface through the polar gaps and leave the light cylinder. The return current $\left(J_{i}\right)$ is created by the outergap where $e^{ \pm}$pairs are produced and separated by local electric field. The existence of the outergap can maintain the current flow of the pulsar magnetosphere (see Fig. 1). This outergap is shown to exist far from the star, where the rotating speed of the corotating magnetosphere is a significant fraction of the speed of light. The observed $\gamma$-ray from the $\mathrm{Crab}$ and Vela pulsars are emitted from this region. The detail spectrum of $\gamma$-ray depends upon the parameters of pulsars, namely, the stellar rotation period $(P)$ and the surface magnetic field $\left(B_{s}\right)$.

According to outergap model, the pair production and radiation mechanisms in the outergap for the Crab pulsar and the Vela pulsar are very different, the former is called the Crabtype outergap and the latter is the Vela-type outergap. Also, the calculation of the model $\gamma$-ray spectrum of the Crab pulsar cannot be done analytically (CHR II; Ho 1989). This makes the analysis of the evolution of the $\gamma$-ray spectrum difficult. On the other hand, the model $\gamma$-ray spectrum of the Vela pulsar can be evaluated analytically; therefore it is possible to study the features and evolution of the $\gamma$-ray spectra for those pulsars with pulsar parameters similar to those of the Vela pulsar which are called Vela-like pulsars. Ruderman \& Cheng (1988) have used the outergap models with an assumption that the mean distance to the outergap only depends on the period of 


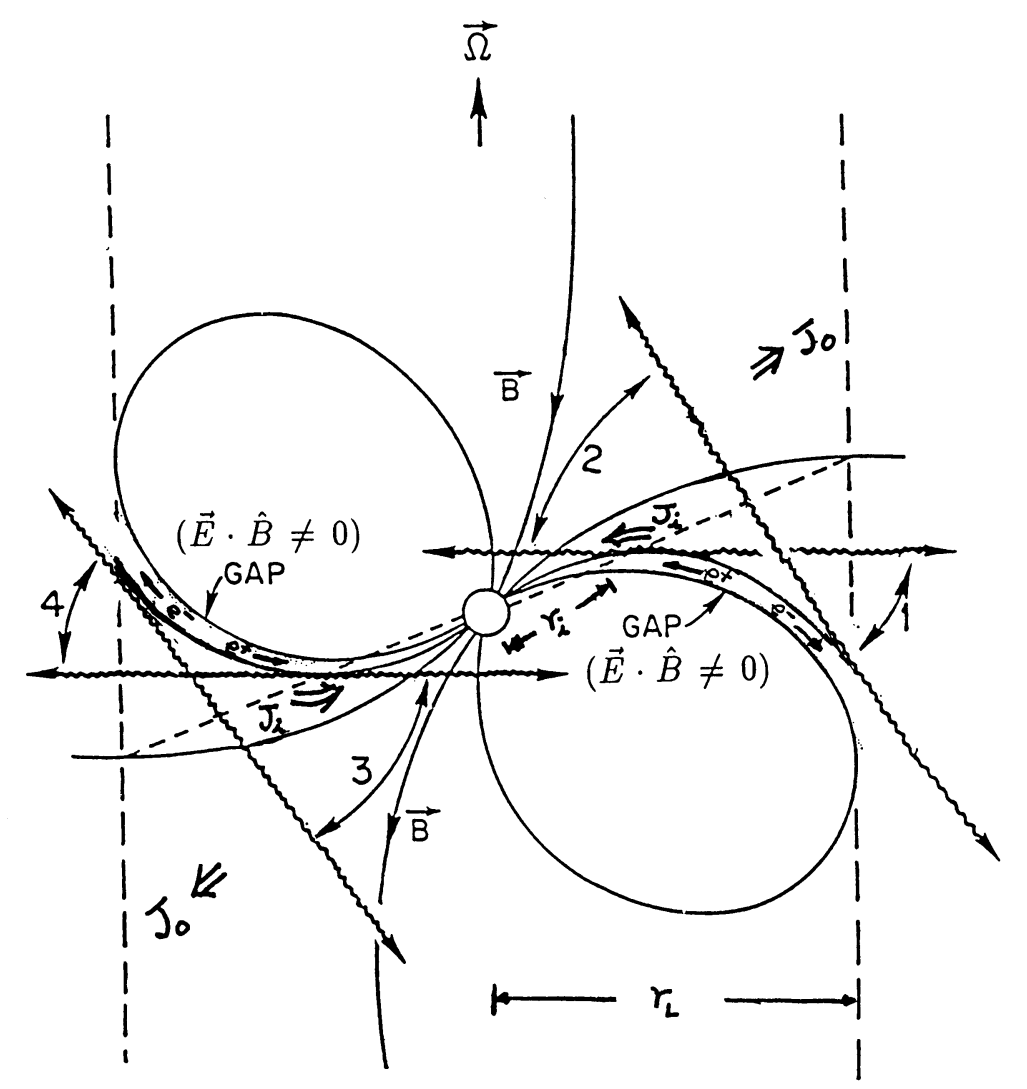

Fig. 1.- Schematic illustration of the location and geometry of outergaps, the current flow pattern in the magnetosphere and the radiation beaming of secondary radiation from four emission regions. The outergaps, where $\boldsymbol{E} \cdot \widehat{\boldsymbol{B}} \neq 0$, start at $r_{i}$ where the null surface intersect the last close field lines and extend to $r_{L}$.

the star and ignores the fact that such distance also depends on the inclination angle of the pulsar to analyze the evolution of outergap. They conclude that the Vela pulsar should turn off as a $\gamma$-ray emitter between $0.1-0.2 \mathrm{~s}$.

Recently, Chen \& Ruderman (1993) motivated by the discovery of Geminga as a $\gamma$-ray pulsar reexamine the death line of the outermagnetospheric accelerator by taking into account the effect of inclination. They argue that the mean distance to the outergap from the star will depend on the starting position of the outergap, where is the intersection between null charge surface and the first open field lines, which is determined by the inclination angle. Therefore, pulsars with the same surface magnetic field but large inclination angle can survive as $\gamma$-ray pulsar longer than those with smaller inclination angle.

In this paper, we will use the outergap model (CHR I; CHR II) and the idea proposed by Chen \& Ruderman (1993) to investigate the evolution of the $\gamma$-ray spectra of the short period pulsars with various inclination angles. We want to point out that only the effect of inclination cannot explain the differences of $\gamma$-ray spectrum of the five-observed $\gamma$-ray pulsars. The variation of mean free path of the primary photons must be also included. In $\S 2$, we summarize some important results of the outergap model. In $\S 3$, we modify the outergap results by taking the variations of inclination angle and photon mean free path into account. In $\S 4$, we describe how the relative intensity and phase separation of pulses depend on the viewing angle. In $\S 5$, we explain why older pulsars are more efficient $\gamma$-ray emitters. In $\S 6$, we use our model to fit the observed spectra of $\gamma$-ray pulsars and discuss the implications of the best fit parameters on gamma ray efficiency, relative intensity and phase separation of pulses. Finally, we present our conclusion in $\S 7$.

\section{REVIEW OF OUTERMAGNETOSPHERIC GAP MODEL}

Cheng, Ho, \& Ruderman (1986a, b) constructed a semianalytical outer magnetospheric gap model of rapidly spinning neutron stars. They assumed that a global current flow pattern through the magnetosphere of a rapidly spinning magnetized neutron star results in large regions of magnetospheric charge depletion (gaps). This would result in a large electric field along the magnetic field lines $(\boldsymbol{E} \cdot \hat{\boldsymbol{B}} \neq 0)$ in those regions, which, through various mechanisms, including inverse Compton scattering and photon-photon pair production, would sustain enough $e^{ \pm}$pair production to:

1. short out $\boldsymbol{E} \cdot \widehat{\boldsymbol{B}}$ except in an almost slablike volume (the "outergap") and

2. maintain the huge magnetospheric current flow.

According to this model, the detail pair production and radiation mechanisms of the Vela-type outergap (the conditions of pulsars with Vela-type outergap will be discussed later) are following. The members of paired $e^{ \pm}$are created within the gap (primary pairs) and accelerated in opposite direction to extreme relativistic energies. The primary $e^{ \pm}$produce $\gamma$-ray (primary) through inverse Compton scattering on IR photons. Here primary $\gamma$-ray are sufficiently energetic to produce $e^{ \pm}$ pairs (secondary) in collision with the same IR photon flux; synchrotron radiation of these secondary pairs gives crossed fan beams of secondary $\gamma$-ray and weaker one of X-ray. Colli- 
sions of the secondary $\gamma$-ray and X-ray produce a large flux of lower energy $e^{ \pm}$pairs (tertiary), much further from the outergap, which fill much of the outermagnetosphere. It is the (tertiary) IR synchrotron radiation from tertiary pairs through the outergap which causes the initial primary inverse Compton scattering in the gap and converts the scattered $\gamma$-ray, thus initiating the entire series of pair production processes. Figure 1 schematically shows the location of outergap and the radiation beaming of secondary radiation from four emission regions.

The pair production and radiation mechanisms of the Velatype outergap and the Crab-type outergap are quite different. The primary $e^{ \pm}$of the Crab pulsar lose most of their energies via curvature radiation instead of synchrotron radiation as in the case of the Vela pulsar. As a result, the power of the Crablike and Vela-like pulsars have different parametric dependence on $P$ and $B_{s}$. The radiation power loss of the Crab-like pulsars is

$$
L_{\gamma} \simeq 1.5 \times 10^{37} \mathrm{P}_{\mathrm{ergs} \mathrm{s}}^{-1} .
$$

In calculating the $\gamma$-ray spectrum of the Vela pulsar, Cheng et al. (CHR I; CHR II) have made some simplifying assumptions. They argue that the secondary $e^{-} / e^{+}$production distribution in the Vela-type outergap would scales like $\gamma^{-1}$, where $\gamma$ is the $e^{-} / e^{+}$Lorentz factor and most of the secondary $e^{ \pm}$have the similar pitch angle, $\theta$, with respect to the local magnetic field, $B$. Then the steady state distribution is roughly given by (Blumenthal \& Gould 1970):

$$
N_{e}(\gamma) \sim \gamma^{-2} \ln \left(\frac{\gamma_{\max }}{\gamma}\right),
$$

where $\gamma_{\max }$ is the maximum energy of the primary $e^{-} / e^{+}$which is determined by the fact that the energy of the primary photons must be just enough to make pairs with the IR photons with a typical energy, $E_{\mathrm{IR}}$, therefore, $\gamma_{\max } \sim m c^{2} / E_{\mathrm{IR}}$. The typical energy of the IR photons is estimated to be (CHR II)

$$
E_{\mathrm{IR}} \sim \frac{\hbar}{\omega_{B}^{3}}\left(\frac{m c^{3} \Omega}{e^{2}}\right)^{2},
$$

where $\omega_{B}=(e B / m c), B(r)=B_{s} R^{3} / r^{3}$ for a typical dipole field, $r$ is the typical distance to the outergap and $R$ is the stellar radius. Because the maximum energy of secondary $e^{ \pm}$is $\gamma_{\max } m c^{2}$, the typical energy of the synchrotron photons from the secondary $e^{ \pm}$is

$$
E_{\max } \sim \frac{3}{2} \gamma_{\max }^{2} \sin \theta \hbar \omega_{B} \propto \Omega^{-4} B^{7}
$$

where $\theta$ is the mean pitch angle of the secondary pairs with respect to the local magnetic field. If the primary photons are emitted tangential to the local field then $\sin \theta \approx\left(1 / \gamma_{\max }\right)+$ $(\lambda / S) \sim \lambda / S$ where $\lambda$ is the mean free path of the primary photons and $S$ is the radius of curvature of the local magnetic field lines. In general, $\sin \theta$ should vary from pulsar to pulsar (see Table 1). For $E_{\gamma}>E_{\max }$, the photon flux will drop exponentially, so this energy is regarded as an upper spectral cutoff. Because of the weakness of the magnetic field in the outer magnetosphere of Vela-type pulsars, an relativistic $e^{-} / e^{+}$does not radiate away all of its energy through synchrotron loss before it leaves the light cylinder. It is argued that once electrons (or positrons) leave the light cylinder, they cannot retain the memory of the neutron star rotation and the radiation beyond the light cylinder will not be pulsed (Kwok, Cheng, \& Lau 1991; Cheung \& Cheng 1993). Thus, the distribution of secondary pairs given by equation (2) is valid only for $\gamma>\gamma_{\min }$, where $\gamma_{\min }$ is roughly given by

$$
\gamma_{\min } \sim \frac{\Omega m c^{3}}{e^{2} \omega_{B}^{2} \sin ^{2} \theta}
$$

Such $\gamma_{\min }$ corresponds to a lower energy spectral break in the photon spectrum which is given by

$$
E_{\min } \sim \frac{3}{2} \gamma_{\min }^{2} \sin \theta \hbar \omega_{B} \propto \Omega^{2} B^{-3}
$$

Therefore, the distribution of the secondary pairs can be approximated by

$$
N_{e}(\gamma) \sim \begin{cases}\gamma^{-2} \ln \left(\gamma_{\max } / \gamma\right), & \gamma_{\min } \leq \gamma \leq \gamma_{\max } \\ 0, & \gamma<\gamma_{\min } .\end{cases}
$$

Here, we have assumed that no $e^{-} / e^{+}$is created below $\gamma_{\min }$

The pulsed radiation spectrum from a Vela-type outergap is calculated by using the $e^{ \pm}$pair distribution of equation (7) with the single particle synchrotron radiation spectrum,

$$
\frac{d^{2} N_{\gamma}}{d E_{\gamma} d t} \propto \frac{1}{E_{\gamma}} \int_{\gamma_{\min }}^{\gamma_{\max }} d \gamma N_{e}(\gamma) F(x),
$$

where $F(x)=x \int_{x}^{\infty} K_{5 / 3}(y) d y$ with $K_{5 / 3}$ is the modified Bessel function, whose values and asymptotic form are given by Ginzberg \& Syrovatiskii (1965), and $x=E_{\gamma} / E_{\text {syn }}$ and $E_{\text {syn }}=3 \gamma^{2}$ sin $\theta \hbar \omega_{B} / 2$. The normalization of equation (8) is determined by the fact that the $\gamma$-ray efficiency of the Vela-type outergap defined by

$$
\eta=\frac{L_{\gamma}}{I \Omega \AA}
$$

is almost a constant which is insensitive to $B_{s}$ and $P$ (for the Vela pulsar $\eta \sim 6 \%$ ). We can estimate when pulsars start to switch from the Crab-type outergap to the Vela-type outergap by equating equations (1) and (9) which give the transition

TABLE 1

The Best-Fit and Deduced Parameters of Five $\gamma$-Ray Pulsars

\begin{tabular}{cccccccrrr}
\hline \hline Pulsar & Period (s) & $B_{s 12}(\mathrm{G})$ & $\sin \theta$ & $\alpha$ & $E_{\min }(\mathrm{eV})$ & $E_{\max }(\mathrm{eV})$ & $\eta^{\mathrm{a}}$ & $P_{c}(\mathrm{~s})$ & $\chi^{2}$ \\
\hline Vela $\ldots \ldots \ldots \ldots \ldots \ldots \ldots$ & 0.089 & 3.37 & $10^{-3}$ & 0.77 & $1.1 \times 10^{7}$ & $7.5 \times 10^{9}$ & 5 & 0.121 & 47.7 \\
PSR 1706 - $44 \ldots \ldots \ldots \ldots$. & 0.1024 & 3.13 & $10^{-3}$ & 0.54 & $1.6 \times 10^{6}$ & $7.1 \times 10^{11}$ & 6 & 0.183 & 3.6 \\
PSR 1509 $-58 \ldots \ldots \ldots \ldots$. & 0.1502 & 15.43 & 0.04 & 1.22 & $4.6 \times 10^{3}$ & $1.1 \times 10^{8}$ & 13 & 0.232 & 2.4 \\
PSR 1055-52 $\ldots \ldots \ldots \ldots$. & 0.1971 & 1.08 & $2.2 \times 10^{-4}$ & 0.28 & $1.0 \times 10^{9}$ & $1.1 \times 10^{9}$ & $\sim 100$ & 0.211 & 11.1 \\
Geminga $\ldots \ldots \ldots \ldots \ldots \ldots$ & 0.237 & 2 & $3.5 \times 10^{-4}$ & 0.29 & $1.8 \times 10^{8}$ & $3.2 \times 10^{9}$ & 61 & 0.280 & 14.2 \\
\hline
\end{tabular}

${ }^{\mathrm{a}} \eta \equiv L_{\gamma} / I \Omega \dot{\Omega} \times 100 \%$ 
period, $P_{t}$,

$$
P>P_{t}=4.6 \times 10^{-2} B_{12}^{2 / 5} \mathrm{~s} .
$$

Finally, there are three approximation forms for equation (8), namely,

$$
\frac{d^{2} N_{\gamma}}{d E_{\gamma} d t} \sim \begin{cases}\exp \left(-E_{\gamma} / E_{\max }\right), & \text { for } E_{\gamma}>E_{\max }, \\ E_{\gamma}^{-3 / 2} \ln \left(E_{\max } / E_{\gamma}\right), & \text { for } E_{\min }<E_{\gamma}<E_{\max }, \\ E_{\gamma}^{-2 / 3}, & \text { for } E_{\gamma}<E_{\min } .\end{cases}
$$

\section{OUTERGAPS WITH VARIOUS INCLINATION ANGLES}

From equations (4) and (6), we can see that the spectral breaks are quite sensitive to the mean magnetic field of the outergap which equals $B_{s}(R / r)^{3}$ for a dipolar field where $B_{s}$ is the surface magnetic field and $r$ is the mean distance to the outergap (Chen \& Ruderman 1993). In Figure 1, we can see that the outergap extends from an inner boundary $r_{i}$, where the last closed field line intersects the null charge surface, to $r_{L}$, where the corotation speed equals speed of light (N.B. $r_{i} \leq r_{f}$, where the null surface intersects the light cylinder). In a dipolar field approximation, $r_{i} \approx 4 r_{L} / 9 \tan ^{2} \chi($ for $\tan \chi \gg 1)$, where

$$
\chi=\cos ^{-1} \frac{\mathbf{\Omega} \cdot \boldsymbol{\mu}}{|\mathbf{\Omega}||\mu|}
$$

and $\boldsymbol{\mu}$ is the magnetic moment of the star, and $r_{f}=r_{L} / \sin \theta_{0}$, where

$$
\theta_{0}=\tan ^{-1}\left(\frac{3 \tan \chi+\sqrt{9 \tan ^{2} \chi+8}}{2}\right)
$$

is the polar angle of the null charge surface. Since the secondary pairs are confined in the open field lines region, so the range of $r$ is between $r_{i}$ and $r_{f}$. We can characterize $r \equiv \alpha r_{L}$, where $\alpha$ can be ranging from $2 \times 10^{-4} P^{-1}$ to 1.22 for an orthogonal rotator to an aligned rotator. Taking this factor into account, the upper spectral break is given by

$$
E_{\max }=9 \times 10^{5} \alpha^{-21} P_{-1}^{-17} B_{12}^{7} \sin \theta \mathrm{eV},
$$

and the lower spectral break is given by

$$
E_{\min }=11 \alpha^{9} P_{-1}^{7} B_{12}^{-3} \sin ^{-3} \theta \mathrm{eV},
$$

where $P_{-1}$ is the rotation period in units of $0.1 \mathrm{~s}$ and $B_{12}$ is the surface magnetic field in units of $10^{12} \mathrm{G}$.

In principle, if $\chi$ is known, then the spectral features of $\gamma$-ray are determined. It has been argued that $\chi$ can be determined by the pulse width of the radio wave and the polarization properties (Lyne \& Manchester 1988). Although such approach is quite reasonable, it is still difficult to know if this deduced inclination angle is an exact one for a particular pulsar. Therefore, we will treat $\alpha$ as a free parameter with the constraints $2 \times 10^{-4} P^{-1} \leq \alpha \leq 1.22$.

\section{RELATIVE INTENSITY AND PHASE SEPARATION OF PULSES}

In addition to the spectral features, the phase separation between two pulses and their relative intensity can provide useful information on the location of emission regions and the viewing angle. For the geometry of outergap, there are four beams emitted from the gaps. Each approximately covers $90^{\circ}$ in latitude extension if the outergap is confined in very thin slab (see Fig. 1). However, the beam intensity should not be uniform over $90^{\circ}$. Let us take beam 1 as an example to illustrate the argument. Figure $2 a$ sketches the intensity of the
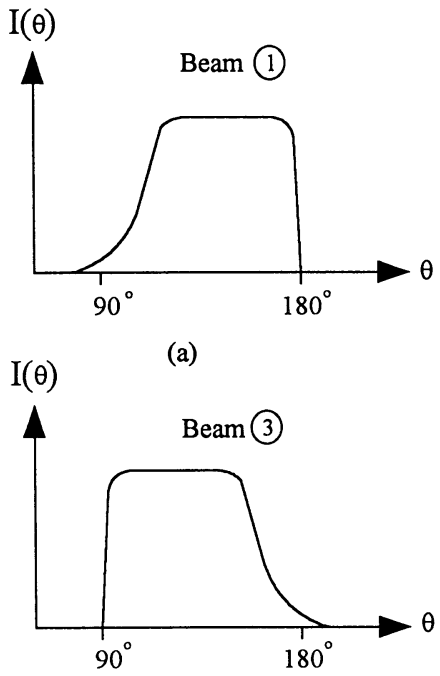

(c)

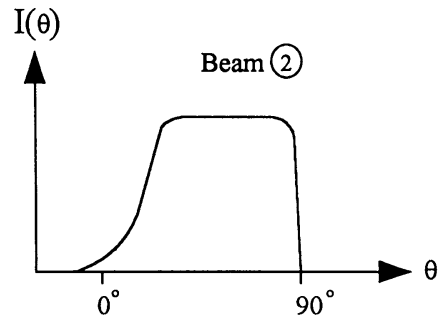

$\mathrm{I}(\theta)$

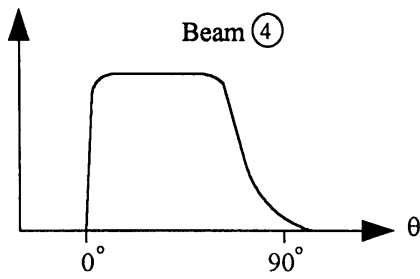

(d)
Fig. 2.-Sketch of the angular distribution of intensity of four beams

beam as a function of polar angle (the spin-axis is chosen to be the z-axis). The gap starts at the null surface. The secondary pairs, which emit the $\gamma$-rays, are gradually increasing toward the light cylinder and eventually reach a steady state. Similarly, we can argue that the intensity of beams 2,3 , and 4 as function of polar angle $(\theta)$ is shown in Figures $2 b-2 d$.

The observed phase separation between two pulses is determined by

1. the flight path difference between the two beams;

2. aberration of the emitted beam direction; and

3. the magnetic field line bending near the light cylinder.

Ignoring factor (3) and using a monopole magnetic field structure, the phase separation is given by (CHR I)

$$
\Delta \varphi=\frac{2}{\pi}\left[\tan ^{-1}\left(\frac{\gamma v}{\sin \theta}\right)+\frac{v}{\gamma} \sin \theta\right]
$$

where $\theta$ is the polar angle of the $\gamma$-ray beam, $\gamma=\left[1-\left(v^{2}\right)\right.$ $\left.\left.c^{2}\right)\right]^{-1 / 2}$ and $v=r \Omega / c$, where $r$ is the distance from the star to the emission region. The quantity $\Delta \varphi$ is ranging from $0^{\circ}$ to $240^{\circ}$ for various $\theta$ and $r$ (see Fig. 9 of CHR I for details). Figures $3 a-3 d$ sketch the phase separation and the relative intensity of pulses as function of the viewing angle $\beta \sim \theta$.

\section{EVOLUTION OF OUTERGAP}

Ruderman \& Cheng (1988) have argued that when the star slows sufficiently down that the upper spectral break will reach the lower spectral break, then it would no longer be possible to maintain the bootstrapped production of $e^{ \pm}$pairs in a pulsar magnetosphere. By equating equations (12) and (13), they conclude that this self-sustained outer magnetosphere accelerator should be terminated when

$$
P=P_{c}=0.17 B_{12}^{5 / 12} \alpha^{-5 / 4} \sin ^{1 / 6} \theta \mathrm{s} .
$$

Therefore, the Vela-type pulsar should have a period which satisfies

$$
4.6 \times 10^{-2} B_{12}^{2 / 5} \mathrm{~s}<P<0.17 B_{12}^{5 / 12} \alpha^{-5 / 4} \sin ^{1 / 6} \theta \mathrm{s} .
$$

Furthermore, key mechanisms which sustain the $e^{ \pm}$pair production needed for Vela's outer magnetospheric current 


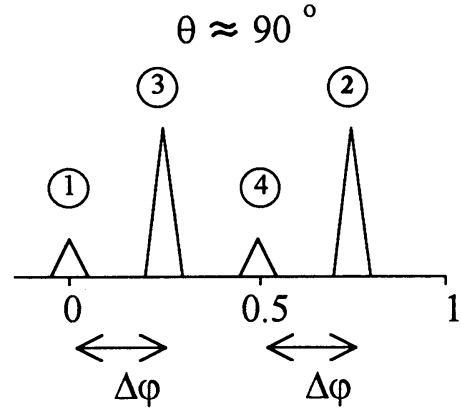

(a)
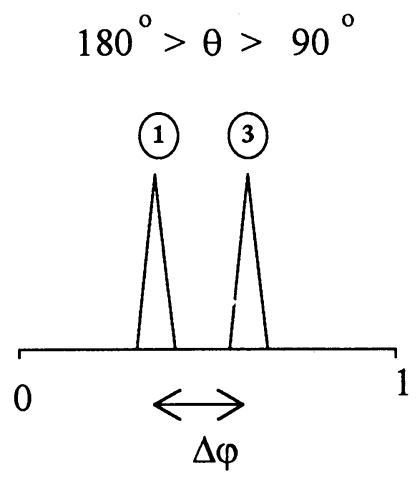

(c)

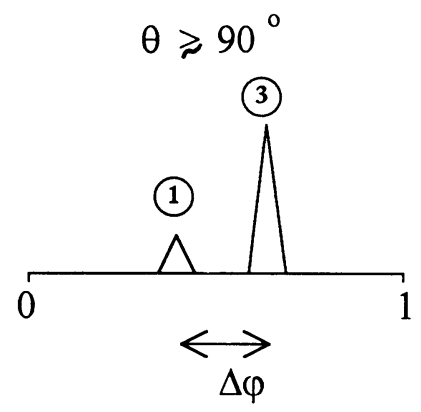

(b)

$\theta \approx 180^{\circ}$

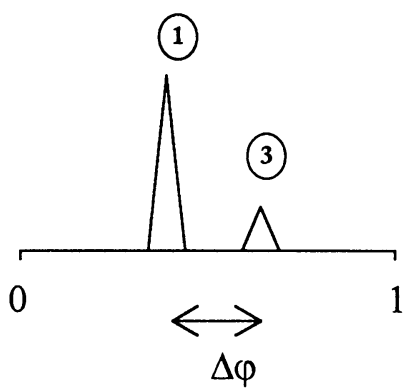

(d)

FIG. 3.- Relative intensity and phase separation of pulses for various viewing angle $\beta \sim \theta . \Delta \varphi$ is given by eq. (14).

flow include $\gamma+\gamma \rightarrow e^{ \pm}$by crossing radiation beams. Pair production for such crossed beam photon collisions is the largest for photon center of mass energy between the threshold $2 m c^{2} \sim \mathrm{MeV}$ and several $\mathrm{MeV}$. As a pulsar's period increases to approach $P_{c}$, the lower spectral break clearly exceeds several $\mathrm{MeV}$, the number of $\gamma$-ray in the beams with energies in the most effective range to make $e^{ \pm}$pairs greatly decreases. Moreover, the suppression of the lower end of $\gamma$-ray spectrum leaves much fewer $\gamma$-ray photons with suitable energies in the beams. Because $E_{\min }$ rises so sharply with increasing $P$, a very much larger fraction of the pulsar's spin down power must then be devoted to making outermagnetospheric pairs and thus to the $\gamma$-ray associated with that $e^{ \pm}$pair production. In Vela the outermagnetosphere accelerator occupies around $\frac{1}{3}$ the total open field line volume. As $P$ increases even very modestly, that fraction must grow very considerably in order to maintain required outer magnetosphere $e^{ \pm}$production until finally all of the available volume is used for the accelerator. Therefore, almost all of the spin down power will be dissipated in $\gamma$-rays:

$$
L_{\gamma} \rightarrow I \Omega \dot{\Omega} \quad \text { as } \quad P \rightarrow P_{c} .
$$

\section{APPLICATIONS}

In this section, we shall investigate those pulsars satisfying equation (16), whose $\gamma$-ray spectral features will be described by equations (11)-(13). In order to obtain a best-fit curve, we define

$$
\chi^{2}=\sum_{i} \frac{\left[\log F_{\gamma}^{\mathrm{th}}\left(\alpha, \sin \theta, E_{\gamma}^{i}\right)-\log F_{\gamma}^{\mathrm{obs}}\left(E_{\gamma}^{i}\right)\right]^{2}}{\sigma_{i}^{2}}
$$

where $\sigma_{i}$ is the observed error bar at $E_{\gamma}^{i}$, and $F_{\gamma}^{\text {th }}$ and $F_{\gamma}^{\text {obs }}$ are the model flux and the observed flux, respectively. The best values of $\alpha$ and $\sin \theta$ are obtained by minimizing $\chi^{2}$. Figures 4-8 compare some model curves with the observed data (Vela, PSR 1706-44, PSR 1055-52, PSR 1509-58, Geminga). The solid line is the best-fit curve, the dotted line and the dashed line are the referenced curves which demonstrate how much $\alpha$ and $\sin \theta$ must be changed so that the model curves cannot fit the observed data. We can see that these two parameters must be off the best-fit values by at least $25 \%$ for $\alpha$ and a factor of 4 for $\sin \theta$, then the model curves become unacceptable. The

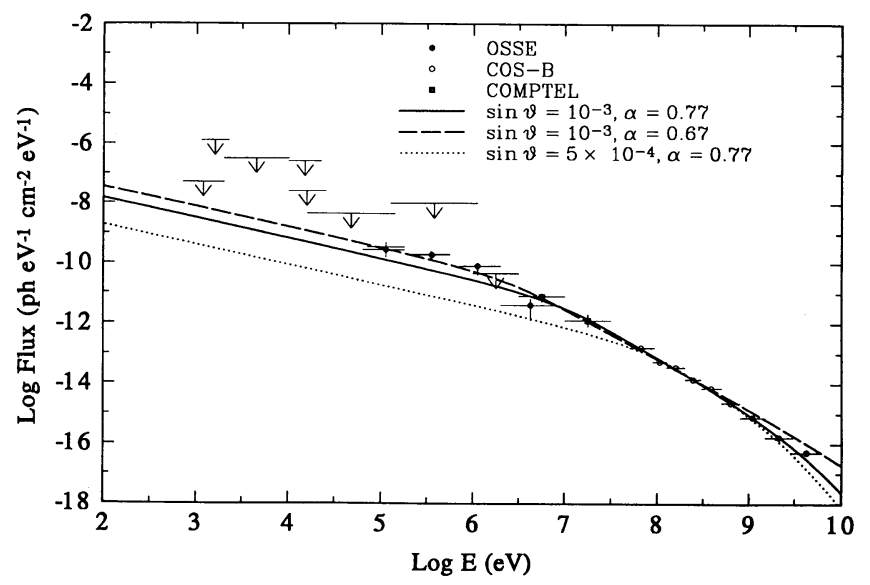

Fig. 4.-Model curves of the Vela pulsar. The observed data include OSSE (Strickman et al. 1992), COMPTEL (Busetta et al. 1992) and COSB (Grenier et al. 1988). 


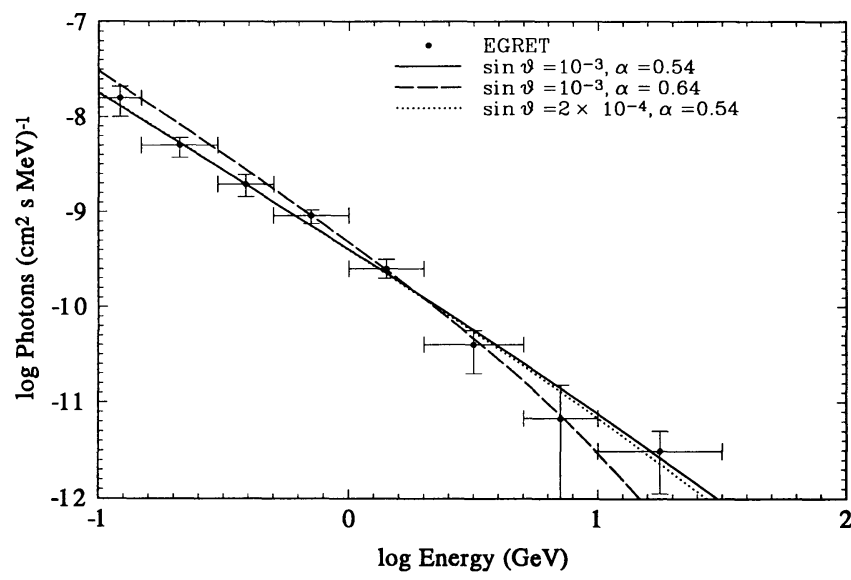

Fig. 5.-Model curves of the pulsar PSR 1706-44. The observed data are from EGRET (Thompson et al. 1992).

model curves are clearly more sensitive to the change of $\alpha$ (see eqs. [12] and [13]). It is interesting to point out some common features of the best-fit curves of these five pulsars:

1. Both free parameters, $\alpha$ and $\sin \theta$, of the best-fit curves satisfy the constraints, namely, $2 \times 10^{-4} P^{-1} \leq \alpha \leq 1.22$ and $1 / \gamma_{\max } \leq \sin \theta \leq 1$, where $\gamma_{\max }$ is the maximum Lorentz of the secondary pairs (see eq. [4]).

2. The spectra of Vela, PSR 1706-44, PSR 1055-52, and Geminga can be fitted by a similar pitch angle $\left(\sin \theta \sim 10^{-3}\right)$. Such small pitch angle implies a very thin radiation region.

3. The present period of Vela, PSR 1706-44, and PSR $1509-58$ are about $60 \%-70 \%$ of their expected turn-off period $\left(P_{c}\right)$. Their $\gamma$-ray efficiency are about $6 \%-15 \%$ of their spindown power. These results do not conflict with equation (9). Furthermore, the period of Geminga and PSR 1055-52 are $86 \%$ and $96 \%$ of $P_{c}$ and their $\gamma$-ray efficiency are near unity which is consistent with equation (17).

4. These $\gamma$-ray pulsars except PSR $1509-58$ are not strong low-energy $\gamma$-ray emitters because of $E_{\min }>\mathrm{MeV}$ (see Table 1).

Since the phase separation and relative intensity of pulses are function of the distance from the star to the emission region which depends on the viewing angle and likely such distance is closed to the mean distance to the outergap. Hence, $\alpha$ gives an

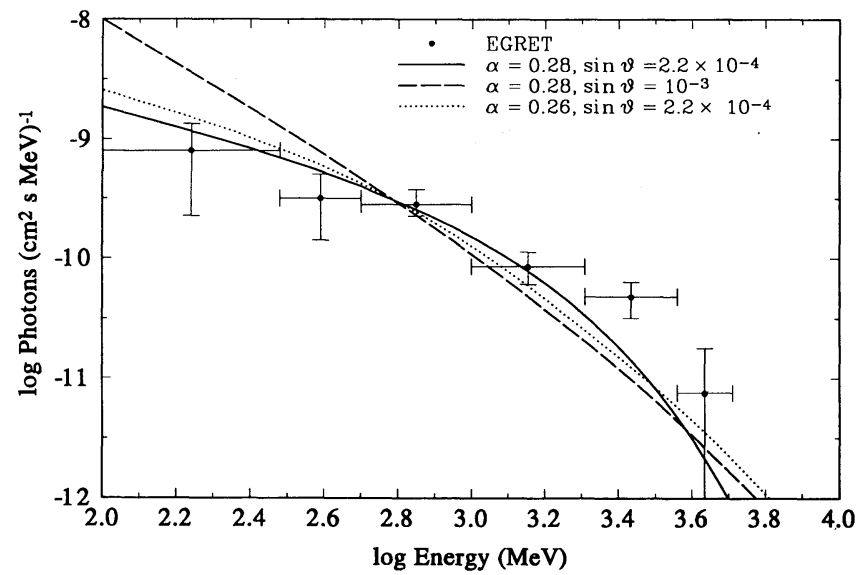

Fig. 6.--Model curves of the pulsar PSR 1055-52. The observed data are from EGRET (Fierro et al. 1993).

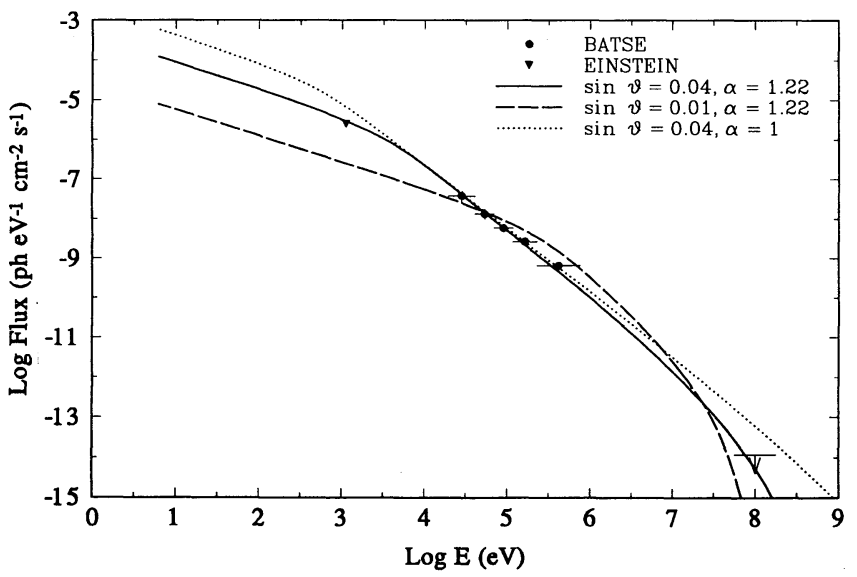

FIG. 7.-Model curves of the pulsar PSR 1509-58. The observed data include Einstein (Seward \& Harden 1982) and BATSE (Wilson et al. 1992).

estimate of the phase separation and the relative intensity of pulses. Let's examine the best-fit parameters, which are listed in Table 1, and the observed data.

1. Vela.-If we assume that the distance to the observed emission region, $r$, equals $\alpha r_{L}$, then $140^{\circ}$ or $220^{\circ}$ phase separation gives $\sim 140^{\circ}$ viewing angle which implies two equal intensity pulses.

2. PSR 1055-52.-This pulsar seems to consist of one single broad pulse or two very closed pulses with phase separation less than $90^{\circ}$ From the best fit $\alpha$, the theoretical phase separation is given by $\Delta \varphi \sim 60^{\circ}-90^{\circ}$, for various $\theta$. It appears in Figure 6 that the dotted line seems to fit the data better than the solid curve; however, its X-ray section is much higher than the observed X-ray (Ogelman \& Finley 1993).

3. PSR 1706-44.- It is not clear that this pulsar emits two pulses or one pulse. If we assume $r=\alpha r_{L}$, then we expect this pulsar emitting double pulses with $\Delta \varphi \sim 120^{\circ}-190^{\circ}$ for various $\theta$. Furthermore, the real value of $\alpha$ for this pulsar is better determined by TeV $\gamma$-ray observation (see Fig. 6). So far the best-fit value of $\alpha$ suggest that this pulsar should emit double pulses and could be a TeV $\gamma$-ray emitters.

4. PSR $1059-58$.- Its $\alpha$ equals 1.22 , which is its upper limit. In other words, the emission region must be very closed to the

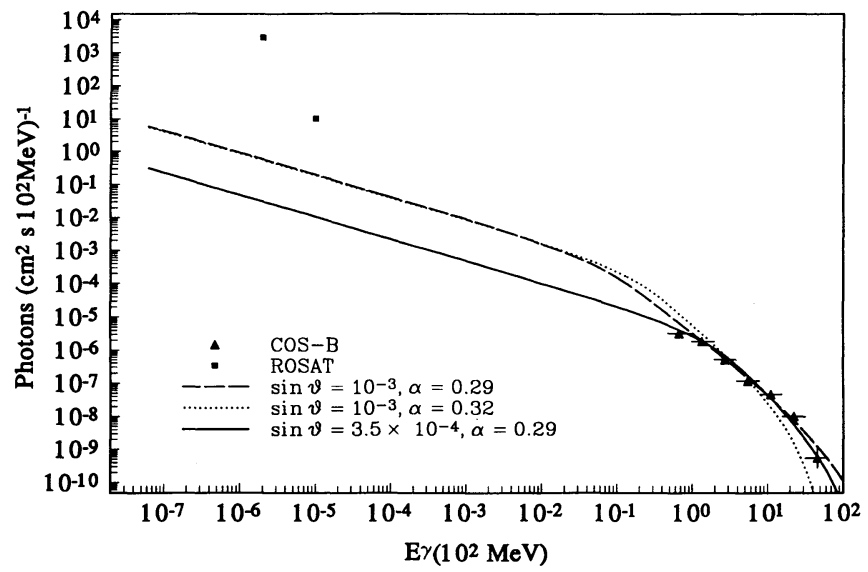

Fig. 8.-Model curves of the Geminga pulsar. The observed data include COS B (Bennett et al. 1977) and ROSAT (Halpern \& Holt 1992). 
light cylinder. So, we are expecting one strong beam and one weak beam which are separated by $180^{\circ}$ that is consistent with the result of OSSE (Ulmer et al. 1993). This also explains why the pitch angle of PSR 1509-58 is much larger than those of the other four pulsars because the radius of curvature of the magnetic field lines near the light cylinder is much smaller than $\left(r r_{L}\right)^{1 / 2}$ due to the drag force of the plasma which becomes very large near the light cylinder. The best-fit curve shows that both $\mathrm{X}$-rays and $\gamma$-rays can be fitted by the same curve which suggests that they are emitted from the same region. Therefore, their pulses should be in phase.

5. Geminga.-Both Geminga and PSR 1055-52 have very small $\alpha$, but Geminga clearly have two equal intensity pulses with $180^{\circ}$ phase separation. This may imply that the viewing angle is close to $90^{\circ}$ which results in two equal strength pulses separated by $180^{\circ}$. We should point out that the model curve is well below the data of ROSAT (Halpern \& Holt 1992). In fact, $\mathrm{X}$-rays are proposed to be thermal X-rays from the stellar surface (Halpern \& Ruderman 1993). It is supported by the fact that the relative phase and light curves of X-ray and $\gamma$-ray are completely different (Becker, Brazier, \& Trümper 1993).

It is difficult to predict the $\gamma$-ray features of other pulsars because $\alpha$ and $\sin \theta$ are two unknown parameters. However, we notice that $\sin \theta$ is always around $10^{-3}$ except PSR $1509-58$. If we focus on those pulsars not close to the terminated period $\left(P_{c}\right)$ whose $\gamma$-ray efficiency is given by equation (9), then some possible model curves can be calculated in terms of $\alpha$. In Table 2 , we extract pulsars satisfying equation (16) with $\alpha=\alpha_{\text {vela }}$ from the Princeton pulsar catalog which contains 558 pulsars. It is interesting to point out that the first three strongest theoretical $\gamma$-ray sources have all been detected (PSR 1055- 52 and Geminga are not in this table because they should have been turned-off as $\gamma$-ray pulsars if they did not have smaller $\alpha$ ). Figure 9 shows the model $\gamma$-ray spectrum of PSR $1951+32$ which is predicted to be the fourth strongest $\gamma$-ray pulsar in Table 2. We have chosen $\sin \theta=10^{-3}$ and $\alpha_{1706} \lesssim \alpha \lesssim \alpha_{\text {Vela }}$. The $\gamma$-ray efficiency is taken to be the mean of Vela and PSR 1706-44. From the theoretical curves, it appears that the $\gamma$-ray of this pulsar can be detected by EGRET, and it should have two pulses with $\Delta \varphi \sim 140^{\circ}-220^{\circ}$.

\section{DISCUSSION}

In this paper, we have used a simplified outergap model to show that the spectrum, $\gamma$-ray efficiency, relative intensity, and phase separation of five known $\gamma$-ray pulsars can be explained in terms of Vela-type outergap model with two free parameters, $\alpha$ and $\sin \theta$. The former characterize the distance to the outergap and the latter is the mean pitch angle of the secondary $e^{ \pm}$pairs. It appears that older pulsars have smaller $\alpha$ which implies larger inclination angle. In fact, this is a deception

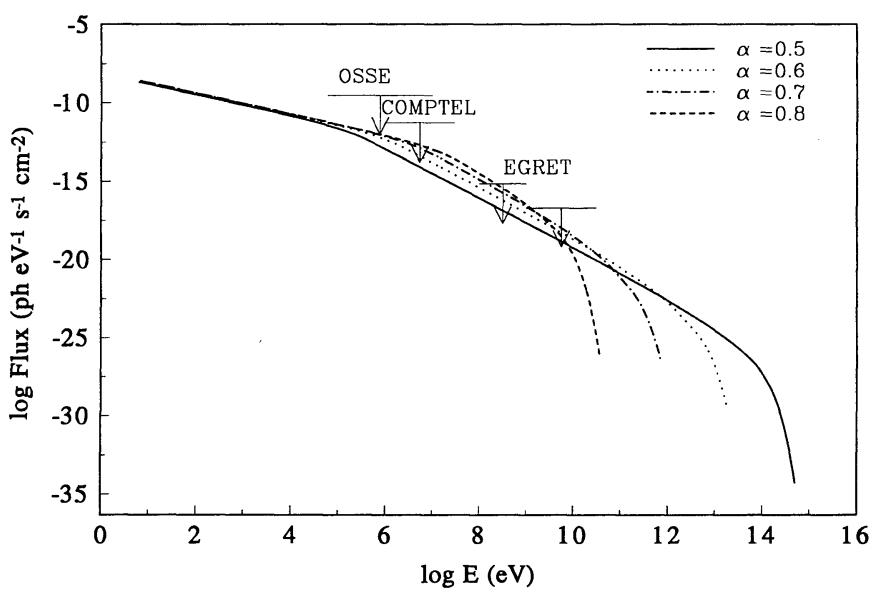

FIG. 9.-Predicted $\gamma$-ray curves of PSR $1951+32$. The arrows are the sensitivities of OSSE, COMPTEL, and EGRET.

because older pulsars with large $\alpha$ will cease as $\gamma$-ray pulsars according to equation (15). Although the inclination angle of pulsars, which can determine the value of $\alpha$, is expected to relate to the pulse width and polarization properties of radio wave, it may not be that accurate for a specific pulsar. The small value of $\sin \theta$ should suggest that the radiation region of outergap is a very thin region. We also want to point out that, in most cases (e.g., Vela, Geminga, PSR 1706-44, PSR $1055-52$ ), when the values of $\alpha$ and $\sin \theta$ are changed by $50 \%$ to $100 \%$, the model curves will not become unacceptable. However, in the case of PSR 1509-58, the curve indeed becomes unacceptable when $\alpha$ is only changed by $25 \%$. It is because the range of the data is from $1 \mathrm{KeV}$ to $100 \mathrm{MeV}$ and our model results indeed quite sensitive to the values of $\alpha$ and $\sin \theta$.

For pulsars (e.g., Vela and PSR 1706-44, also see model curves of PSR $1951+32)$ not closed to the $\gamma$-ray death line, their $\gamma$-ray spectra (spectral indices $\sim 1.7-2.0$ ) in the range of 10 $\mathrm{MeV}-10 \mathrm{GeV}$ seems insensitive to $\alpha$ and $\sin \theta$. The model $\gamma$-ray curves of PSR $1951+32$ could be the real ones if its inclination angle is not as small as that of Geminga and PSR 1055-52. Since the first three strongest sources in Table 2 have been detected as $\gamma$-ray pulsars, it is interesting to see if the rest pulsars may be also $\gamma$-ray pulsars. Their spectral features should be described by equations (11)-(13).

We are grateful to Fulivio Melia for many useful suggestions. This work is supported by the research grant of UPGC of Hong Kong.

TABLE 2

A List of The Vela-TyPe Pulsars With $\alpha=\alpha_{\text {Vela }}$

\begin{tabular}{|c|c|c|c|c|c|}
\hline Pulsar & Period (s) & $B_{s 12}(G)$ & Distance (kpc) & $P_{c}(\mathrm{~s})$ & $I \Omega \Omega / 4 \pi d^{2}\left(\right.$ ergs cm $\left.^{-2} \mathrm{~s}^{-1}\right)$ \\
\hline PSR $0833-45 \ldots \ldots \ldots$ & 0.089 & 3.37 & 0.5 & 0.121 & $2.31 \times 10^{-7}$ \\
\hline PSR $1509-58 \ldots \ldots \ldots$ & 0.150 & 15.4 & 4.4 & 0.222 & $7.74 \times 10^{-9}$ \\
\hline PSR $1706-44 \ldots \ldots \ldots$ & 0.102 & 3.13 & 1.82 & 0.118 & $8.62 \times 10^{-9}$ \\
\hline PSR $1757-24 \ldots \ldots \ldots$ & 0.125 & 4.05 & 4.61 & 0.130 & $1.02 \times 10^{-9}$ \\
\hline PSR $1823-13 \ldots \ldots \ldots$ & 0.101 & 2.79 & 4.12 & 0.112 & $1.40 \times 10^{-9}$ \\
\hline PSR $1951+32 \ldots \ldots \ldots$ & 0.040 & 0.486 & 2.5 & 0.0559 & $5.00 \times 10^{-9}$ \\
\hline PSR $1957+20 \ldots \ldots \ldots$ & 0.0016 & $1.66 \times 10^{-4}$ & 1.53 & 0.00229 & $5.71 \times 10^{-10}$ \\
\hline
\end{tabular}


Ayasli, S., \& Ogelman, H. 1980, ApJ, 232, 227

Becker, W., Brazier, K. T. S., \& Trümper, J. 1994, A\&A, in press

1, Bennett, K., et al. 1977, A\&A, 56, 469

In' Bertotti, B., et al. 1969, Nature, 223, 1351

of Blumenthal, G. R., \& Gould, R. J. 1970, Rev. Mod. Phys., 42, 237

'-I Busetta, M., et al. 1992, in Proc. Isolated Pulsars, ed. K. A. van Riper R. Epstein, \& C. Ho (Cambridge: Cambridge Univ. Press), 236

Chen, K., \& Ruderman, M. A. 1993, ApJ, 402, 264

Cheng, K. S., Ho, C., \& Ruderman, M. A. 1986a, ApJ, 300, 500 (CHR I) 1986b, ApJ, 300, 522 (CHR II)

Cheung, W. M., \& Cheng, K. S. 1993, ApJ, 413, 694

Fierro, J. M., et al. 1993, ApJ, 413, L27

Ginzberg, V. L. \& Syrovatskii, S. I. 1965, ARA\&A 3, 297

Goldreich, P., \& Julian, W. H. 1969, ApJ, 157, 869

Grenier, I. A., Hermsen, W., \& Clear, J. 1988, A\&A, 204, 117

Halpern, J. P., \& Holt, S. S. 1992, Nature, 357, 222

Halpern, J. P., \& Ruderman, M. A. 1993, ApJ, 415, 286

Hardee, P. E. 1979, ApJ, 227, 958

\section{REFERENCES}

Harding, A. K. 1981, ApJ, 245, 267

Hinata, S. 1977, ApJ, 216, 10

Ho, C. 1989 , ApJ, 242, 396

Kwok, P. W., Cheng, K. S., \& Lau, M. M. 1991, ApJ, 379, 653

Lyne, A. G., \& Manchester, R. N. 1988, MNRAS, 234, 477

Ögelman, H., \& Finley, J. P. 1993, ApJ, 413, L17

Ozernoi, L. M.. \& Usov, V. V. 1977, Soviet Astron., 21, 425

Ruderman, M. A.. \& Cheng, K. S. 1988, ApJ, 335, 306

Ruderman M A, \& Sutherland, P. 1975, ApJ, 196, 51

Seward, F. D., \& Harden, F. R. J. 1982, ApJ, 256, 45

Strickman, M., et al. 1992, IAU Circ., No. 5557

Sturrock, P. A. 1971, ApJ, 164, 529

Thompson, D. J., et al. 1992, Nature, 359, 615

Treves, A. 1971, A\&A, 15, 471

Ulmer, M. P., et al. 1993, ApJ, 417, 738

Wilson, R. B., et al. 1992, in Proc. Isolated Pulsars, ed. K. A. van Riper, R. Epstein, \& C. Ho (Cambridge: Cambridge Univ. Press) 